\title{
Guanethidine treatment does not block the ability of central leptin administration to decrease blood glucose concentrations in streptozotocin-induced diabetic rats
}

\author{
Jinpin Wang ${ }^{1}$, Catherine $M$ Wernette ${ }^{1}$, Robert $L$ Judd $^{2}$, Kevin W Huggins ${ }^{1}$ and $B$ Douglas White ${ }^{1}$ \\ Departments of ${ }^{1}$ Nutrition and Food Science and ${ }^{2}$ Anatomy, Physiology and Pharmacology, Auburn University, Auburn, Alabama 36849, USA \\ (Correspondence should be addressed to B D White; Email: whitebd@auburn.edu)
}

\begin{abstract}
Leptin, administered either into the ventricles of the brain or systemically, has been shown to normalize blood glucose concentrations in streptozotocin (STZ)-induced diabetic rats. We hypothesized that an intact sympathetic nervous system is necessary for centrally administered leptin to normalize or attenuate high blood glucose concentrations in STZ-induced diabetic rats. Young male Wistar rats $(\sim 50 \mathrm{~g})$ were treated every other day with either s.c. guanethidine $(100 \mathrm{mg} / \mathrm{kg})$ or vehicle for 2 weeks. Rats were then implanted with an intracerebroventricular cannula directed to the lateral ventricle and made diabetic with an i.v. injection of STZ $(50 \mathrm{mg} / \mathrm{kg})$. Half of the animals in each group were given
\end{abstract}

daily injections of leptin $(10 \mu \mathrm{l})$, while the remaining animals received vehicle injections. Blood glucose concentrations were measured daily and tissue norepinephrine content was determined by high performance liquid chromatography at the end of the study. Guanethidine pretreatment did not block the ability of centrally administered leptin to decrease blood glucose concentrations in diabetic rats. This suggests that the sympathetic nervous system does not mediate the leptininduced attenuation of high blood glucose concentrations observed in diabetic rats.

Journal of Endocrinology (2008) 198, 541-548

\section{Introduction}

Chronic peripheral leptin administration (via subcutaneous osmotic pump) normalizes blood glucose concentrations in streptozotocin (STZ)-induced diabetic rats (Chinookoswong et al. 1999). This is associated with a decrease in the rate of glucose production under basal and hyperinsulinemic conditions, and an increase in whole-body glucose utilization during insulin infusion. Central leptin administration over a period of several days also normalizes blood glucose concentrations in STZ-induced diabetic rats (Hidaka et al. 2002, Lin et al. 2002, da Silva et al. 2006). Several lines of evidence suggest that leptin acts centrally to cause these effects. Central injections of leptin normalize blood glucose concentrations without increasing the serum leptin concentration (Hidaka et al. 2002, Lin et al. 2002), and the dose of leptin that normalizes blood glucose concentrations when injected centrally has no effect when injected systemically (Hidaka et al. 2002). Pair-feeding studies show that the effect of leptin on blood glucose is not secondarily due to a decrease in feeding (Hidaka et al. 2002, Lin et al. 2002). Also, the administration of central leptin does not increase serum insulin concentrations (Hidaka et al. 2002, Lin et al. 2002). In fact, central leptin administration decreases serum insulin concentrations in nondiabetic rats to the level found in
STZ-treated rats (Dobbins et al. 2002, Lin et al. 2002). The mechanism by which central leptin normalizes blood glucose concentrations in STZ-induced diabetic rats is not clear.

One of the reported effects of central leptin is activation of the sympathetic nervous system. Direct nerve recordings have shown that i.v. leptin administration stimulates sympathetic activity in the kidney, adrenal glands, and hind limbs (Haynes et al. 1997). The time course of the response and lack of a doseresponse to circulating leptin concentrations suggest that sympathetic activation by leptin is at least partially mediated by the central nervous system (Haynes et al. 1997). Indeed, lesions of the arcuate nucleus of the hypothalamus abolish the sympathetic response to systemic infusion of leptin (Haynes 2000). Hypothalamic leptin administration increases glucose uptake in heart, brown adipose tissue, and soleus and extensor digitorum longus muscles (Haque et al. 1999). The increase in glucose uptake in these tissues is blocked by prior treatment with guanethidine, suggesting that leptin-mediated glucose uptake into these tissues requires the sympathetic nervous system. Activation of AMP-dependent protein kinase (AMPK) increases glucose uptake in skeletal muscle (Merrill et al. 1997). Intrahypothalamic injections of leptin increase the activity of AMPK in skeletal muscle (Minokoshi et al. 2002). This effect is blocked by surgical disruption of sympathetic innervation to the muscle or by blockade of $\alpha$-adrenergic 
receptors, suggesting that the sympathetic nervous system mediates the central effects of leptin on AMPK activation in muscle (Minokoshi et al. 2002). Infusion of 5-aminoimidazole4-carboxamide-1- $\beta$-D-ribofuranoside (AICAR), a stimulator of AMPK activity, not only increases glucose uptake (Merrill et al. 1997) and oxidation (Smith et al. 2005) in skeletal muscle, but also decreases endogenous glucose production rates (Bergeron et al. 2001). A liver-specific knock out of the AMPK $\alpha 2$ subunit leads to hyperglycemia and a 50\% increase in the rate of endogenous glucose production (Andreelli et al. 2006). Leptin and adiponectin do not affect endogenous glucose production in AMPK $\alpha 2$ knockout mice (Andreelli et al. 2006).

We hypothesized that the leptin-mediated attenuation in blood glucose concentrations of STZ-induced diabetic rats is dependent on an intact sympathetic nervous system. To determine whether the sympathetic nervous system plays a role in mediating the ability of central leptin to reduce blood glucose levels in diabetic rats, we chemically denervated the sympathetic nervous system of a group of rats using guanethidine. A control group received injections of vehicle, rather than guanethidine. All rats were made diabetic by STZ treatment and then received daily intracerebroventricular (i.c.v.) injections of either leptin or vehicle. Blood glucose concentrations were measured daily and the norepinephrine content of various tissues was determined at the end of the study. The results suggest that impairment of the sympathetic nervous system does not alter the ability of central leptin administration to reduce blood glucose concentrations in STZ-induced diabetic rats.

\section{Materials and Methods}

\section{Animals}

Male Wistar rats ( 50 g; Harlan Inc., Prattville, AL, USA) were housed in shoebox cages. Rats were kept in a temperature-controlled room with a 12:12 h light-darkness cycle (lights on $0600 \mathrm{~h}$ ). Rats had free access to chow (Prolab RMH 300, Purina Mills, Richmond, IN, USA) and water. All experimental protocols were approved by Auburn University's Institutional Animal Care and Use Committee.

\section{Experimental design}

Half of the rats were administered guanethidine monosulfate $(100 \mathrm{mg} / \mathrm{kg}$; Sigma-Aldrich Co.) s.c. in the area of the back every other day for 2 weeks. The other half of the rats did not receive guanethidine, but did receive control injections of saline. After 2 weeks of injection, rats were allowed to recover. Body weights were determined every other day during the guanethidine injection and recovery periods, and on select days thereafter (prior to STZ injection and prior to killing). Rats were then implanted with an i.c.v. cannula directed into the lateral ventricle (See Cannula placement) and moved to individual hanging cages. All rats were made diabetic by the administration of STZ (see Induction of diabetes). Once hyperglycemia was verified, rats were administrated either i.c.v. leptin $(10 \mu \mathrm{g}$; Calbiochem, San Diego, CA, USA or R\&D Systems Inc., Minneapolis, MN, USA) or vehicle (a 5:3

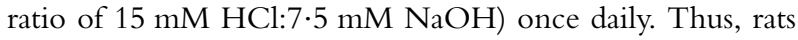
were divided into four treatment groups: vehicle-vehicle, vehicle-leptin, guanethidine-vehicle, and guanethidineleptin. Blood samples were collected daily from the tail vein and blood glucose concentrations were measured (see Blood sampling and glucose determination). In phase 1, i.c.v. leptin was administered daily for 6 consecutive days. After day 6 , i.c.v. leptin injections were stopped for 9 days to determine if the effect of leptin on blood glucose concentrations was reversible. In phase 2, daily i.c.v. leptin injections were reinitiated for an additional 6 days. After the experimental period of 21 days, the rats were anesthetized with pentobarbital, and the spleen, brown adipose tissue, white adipose tissue, and liver were collected to determine norepinephrine content. Norepinephrine measurements were used as an indicator of how well guanethidine treatment decreased sympathetic activity.

\section{Cannula placement}

Rats were anesthetized by an i.p. injection of ketamine and xylazine $(100 \mathrm{mg} / \mathrm{kg}$ and $1 \mathrm{mg} / \mathrm{kg}$ respectively) and placed in a stereotaxic apparatus. A 22 gauge, stainless steel guide cannula (Plastics One, Roanoke, VA, USA) was implanted into the lateral ventricle $(0.8 \mathrm{~mm}$ posterior and $1.4 \mathrm{~mm}$ lateral to bregma, $3.5 \mathrm{~mm}$ ventral to the surface of the skull). Four stainless steel screws and dental cement were used to fix the guide cannula to the skull. A 'dummy' cannula $1 \mathrm{~mm}$ longer than the guide cannula was placed into the guide cannula. The rats were housed in individual cages and allowed to recover for 4 days. Angiotensin II (40 ng in a total volume of $6 \mu \mathrm{l}$; Sigma-Aldrich Co.) was used to verify the accurate placement of the cannula. Rats that drank less than $5 \mathrm{ml}$ of water within $15 \mathrm{~min}$ of administration of angiotensin II were excluded from the experiment. After the drinking test, 31 rats were used in this study.

\section{Induction of diabetes}

Diabetes was induced with a single i.v. injection of freshly prepared STZ (50 mg/kg; Sigma-Aldrich Co.) in $0.05 \mathrm{M}$ citrate buffer $(\mathrm{pH} 4 \cdot 5)$ via the tail vein. Control rats were injected with citrate buffer only. All STZ-injected rats had blood glucose concentrations greater than $350 \mathrm{mg} / \mathrm{dl}$ before the beginning of the leptin injections.

\section{Blood sampling and glucose determination}

Blood was sampled via the tail vein. Rats (in the fed state) were held with their tails immersed in warm water $\left(48^{\circ} \mathrm{C}\right)$ for $30-45$ s to dilate the vein. A syringe with a 26 gauge needle was then used to withdraw a small amount of blood. Blood 
glucose concentrations were determined with an Accu-Chek Active handheld glucometer (Roche Diagnostics).

\section{Determination of tissue norepinephrine and protein content}

Norepinephrine concentrations were measured by high pressure liquid chromatography (HPLC). Tissues were crushed under liquid nitrogen with a mortar and pestle. About $100 \mathrm{mg}$ of sample was placed in pre-weighed microcentrifuge tubes. A cold solution containing $0 \cdot 2 \mathrm{M}$ perchloric acid and $1 \mathrm{ng} / \mathrm{ml}$ ascorbic acid (PCA/AA, $790 \mu \mathrm{l}$ ) was added and this mixture was sonicated thrice $(10-30 \mathrm{~s} \mathrm{each}$ time) on ice. Ten microliters of an internal standard, 3,4dihydroxybenzylamine hydrobromide (Sigma-Aldrich Co.) were added. The solution $(800 \mu \mathrm{l})$ was mixed and centrifuged at $9300 \mathrm{~g}$ for $10 \mathrm{~min}$. The supernatant $(600 \mu \mathrm{l})$ was added to a tube containing $200 \mathrm{mg}$ alumina, which was then mixed with $1 \mathrm{ml} \mathrm{0.5} \mathrm{M} \mathrm{Tris-} \mathrm{HCl}(\mathrm{pH} \mathrm{8} \cdot 6)$ and centrifuged at $9300 \mathrm{~g}$ for $1 \mathrm{~min}$. The supernatant was removed and discarded, and the alumina was washed twice by mixing with $1 \mathrm{ml}$ water followed by centrifugation for $1 \mathrm{~min}$ at $9300 \mathrm{~g}$. After removing all of the supernatant, catecholamines were eluted from the alumina by mixing with $300 \mu \mathrm{lCA} / \mathrm{AA}$ followed by centrifugation for $1 \mathrm{~min}$ at $9300 \mathrm{~g}$. The PCA/AA supernatant $(\sim 300 \mu \mathrm{l})$, which contained the catecholamines, was transferred to vials suitable for HPLC analysis. The tissue extracts and standards $(10 \mu \mathrm{l})$ were processed by HPLC using a Gynkotek HPLC pump (P580: Gynkotek GmbH, Munich, Germany) and HPLC autosampler (Model GINA 50) attached to a Hewlett-Packard Zorbax SB-C18 Stable Analytical Column $(4 \cdot 6 \times 250 \mathrm{~mm}, 5$-micron $)$ pre-equilibrated with citric acid buffer $(21.01 \mathrm{~g}$ citrate, $245 \mathrm{mg}$ sodium octyl sulfate per liter, $10 \%$ ethanol, $\mathrm{pH} 4$ ) at $0.7 \mathrm{ml} / \mathrm{min}$. The samples were analyzed at $50 \mathrm{nA}$ for $15 \mathrm{~min}$ with a Decade digital electrochemical amperometric detector and VT-03 electrochemical flow cell (both from Gynkotek). The data were collected using the Chromeleon chromatography information management system (Gynkotek) and a Gateway PC computer. Protein concentrations were determined by the Bio-Rad protein assay (Bio-Rad Laboratories, Inc). Norepinephrine content was expressed as ng per mg protein.

\section{Statistical analysis}

All results are presented as means \pm s.E.M. Statistical analyses were performed using SPSS 12.0 (SPSS Inc., Chicago, IL, USA) and SAS 8.02 (SAS Institute Inc., Cary, NC, USA) or JMP IN 5.1.2. (SAS Institute Inc). Repeated-measures ANOVA was used to determine the effects of guanethidine and leptin treatment on blood glucose concentrations and the effects of guanethidine treatment on body weight. A one-way ANOVA was used to determine the effect of guanethidine treatment on blood glucose concentrations, prior to making the rats diabetic and after making them diabetic, but prior to central leptin administration. Two-way ANOVA was used to determine the main effects of guanethidine and leptin treatment and their interaction on norepinephrine content within the various tissues. Contrast statements or least-squares Student's $t$-tests were used as a follow-up test to compare individual groups. A difference of $P \leq 0.05$ was considered statistically significant.

\section{Results}

Effects of guanethidine and leptin treatment on body weight

Guanethidine treatment resulted in a decrease in body weight (Fig. 1A). Across the period of guanethidine treatment and
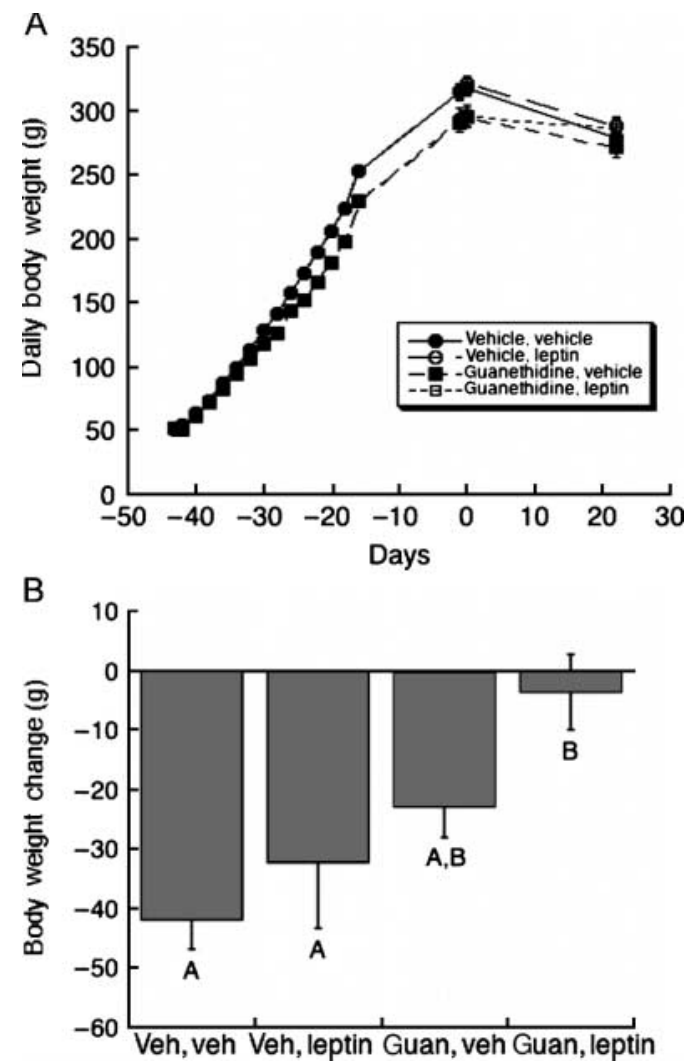

Figure 1 (A) Daily body weights of Wistar rats. Guanethidine $(100 \mathrm{mg} / \mathrm{kg})$ was delivered subcutaneous every other day for 2 weeks (day -42 to day -28 ). Vehicle-treated rats received saline injections. An i.c.v. cannula directed at the lateral ventricle was implanted into each rat beginning on day -15 . All rats were given an i.v. bolus of streptozotocin $(50 \mathrm{mg} / \mathrm{kg})$ on day 0 to induce diabetes. Once hyperglycemia was confirmed, rats in each group (guanethidine-treated versus vehicle-treated) were given daily i.c.v. injections of either leptin $(10 \mu \mathrm{g})$ or vehicle. Rats were killed and tissues were collected on day 22. Results are shown as means \pm s.E.M. Group means represent between 8 and 11 observations. (B) Average change in body weight of rats between the day diabetes was induced (day 0 ) and the completion of the study (day 22). Results are shown as means \pm s.E.M. Group means represent between 6 and 10 observations. Means with different letters indicate statistically significant differences $(P<0 \cdot 05)$. 
recovery, guanethidine-treated rats had lower body weights $(P=0.0006)$ than untreated rats. This became a statistically significant difference by day 12 after guanethidine injections began. The difference in body weights reached $\sim 10 \%$ and was maintained even after guanethidine injections ceased through the time rats were made diabetic by the injection of STZ.

Induction of diabetes caused a significant decrease in body weight $(\sim 40 \mathrm{~g})$ over a period of 3 weeks (Fig. 1B). The diabetes-induced decrease in body weight was significantly attenuated by the prior treatment of guanethidine $(P=0 \cdot 007)$. Leptin treatment appeared to also attenuate the diabetes-induced decrease in body weight, though this did not quite reach statistical significance $(P=0 \cdot 08)$. The combination of prior treatment with guanethidine and daily i.c.v. leptin injections prevented the diabetes-induced decrease in body weight, such that body weight remained essentially unchanged over the 3-week period.

\section{Effect of guanethidine and central leptin on blood glucose concentration}

Before diabetes was induced and immediately after induction (i.e., prior to central leptin administration), there were no differences in blood glucose concentrations between rats previously treated with guanethidine and control animals (Fig. 2). Prior to the induction of diabetes, both groups of rats had blood glucose concentrations around $120 \mathrm{mg} / \mathrm{dl}$. The day after treatment with STZ, the

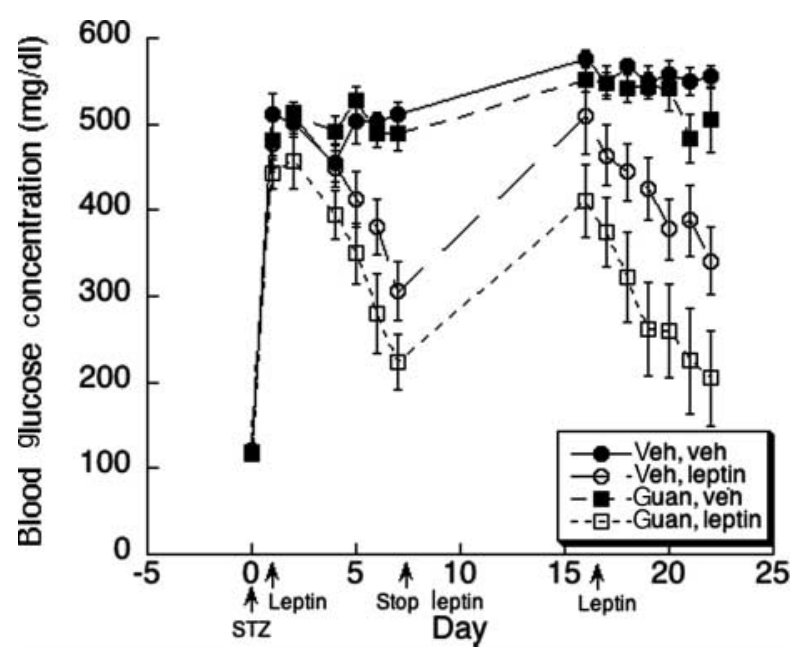

Figure 2 Blood glucose concentrations $(\mathrm{mg} / \mathrm{dl})$ of Wistar rats pretreated every other day with either guanethidine (guan) $(100 \mathrm{mg} / \mathrm{kg})$ or the vehicle (veh) for 2 weeks. Rats were then implanted with a cannula directed into the lateral ventricle, made diabetic with an i.v. injection of streptozotocin $(50 \mathrm{mg} / \mathrm{kg})$, and treated daily with either leptin (10 $\mu$ g) or vehicle (veh). Two sets of daily leptin injections were administered over the experimental period. Results are shown as means \pm s.E.M. Group means represent between 6 and 11 observations. blood glucose concentration of the guanethidine-treated and control rats averaged $460 \pm 18$ and $495 \pm 16 \mathrm{mg} / \mathrm{dl}$ respectively. Daily central leptin administration decreased blood glucose concentrations by day 4 in both guanethidine-treated and control animals. The leptin-induced decrease continued through day 7 . Considering days $4-7$, there was an effect of leptin $(P<0.0001)$, time $(P=0 \cdot 005)$, and an interaction between these two factors $(P<0 \cdot 0001)$. Though there was no interaction between guanethidine and leptin, the guanethidine, leptin-treated rats had lower blood glucose concentrations than control, leptin-treated rats $(P=0 \cdot 014)$ on days 6 and 7. After discontinuing central leptin treatment for 9 days (day 16), blood glucose concentrations increased toward the high levels maintained by the diabetic non-leptin-treated rats. The blood glucose concentrations of control, leptintreated rats increased to levels that were not statistically different from the values obtained from rats previously receiving i.c.v. vehicle injections. However, blood glucose concentrations of guanethidine-treated rats previously treated with leptin were still lower than those of the other groups. The second round of leptin injections again decreased blood glucose concentrations in both vehicleand guanethidine-treated animals. Considering days 16-22, there was a decrease in blood glucose concentrations in rats treated with leptin $(P<0 \cdot 0001)$. There was also an effect of time $(P=0.001)$ and an interaction between the effect of leptin and time $(P=0 \cdot 035)$. In addition, there was an overall effect of guanethidine treatment $(P=0 \cdot 035)$ to lower blood glucose concentrations in leptin-treated animals. Over this 6-day injection period, guanethidine administered leptin-treated rats had lower blood glucose concentrations than did vehicle administered leptin-treated rats $(P=0 \cdot 013)$. During this period, blood glucose concentrations were not different between guanethidine and vehicle previously administered vehicle-treated rats.

\section{Norepinephrine content in tissues}

Guanethidine treatment decreased norepinephrine content in spleen $(P<0 \cdot 001$; Fig. 3), brown adipose tissue $(P<0 \cdot 001$; Fig. 4), and white adipose tissue $(P=0 \cdot 034$; Fig. 5). However, previous treatment with guanethidine did not have an effect on norepinephrine content in the liver (Fig. 6). The average decrease in norepinephrine content due to guanethidine treatment was 65,61 , and $85 \%$, for the spleen, brown adipose tissue, and white adipose tissue respectively. In the liver, there was an overall effect for norepinephrine content to be greater in leptin-treated rats than in rats not treated with leptin $(P=0 \cdot 001)$. An interaction between guanethidine and leptin treatments that approached statistical significance $(P=0 \cdot 07)$ suggests this result was mostly due to the effect of leptin in guanethidine-treated rats. Daily leptin treatment had no significant effect on norepinephrine content in any other tissue tested. 


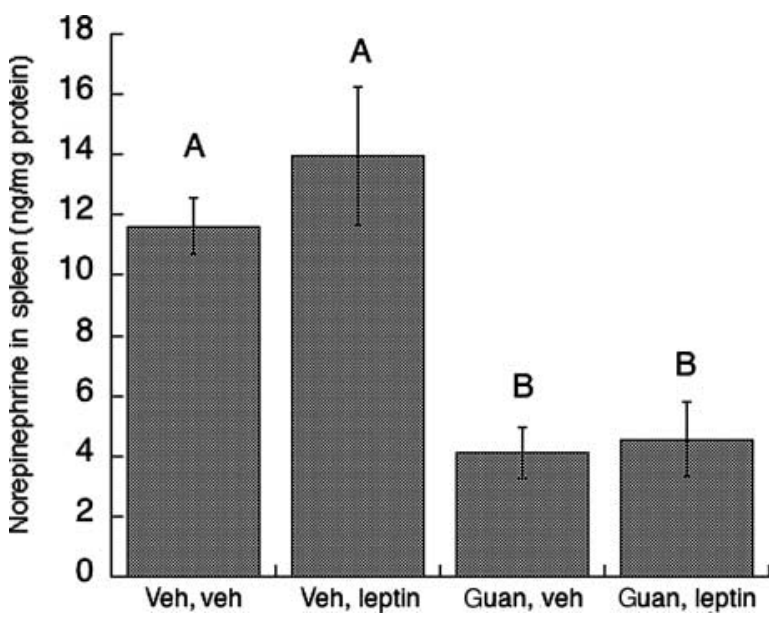

Figure 3 Norepinephrine content in the spleen (ng/mg protein). Wistar rats were pretreated every other day with either guanethidine (guan) $(100 \mathrm{mg} / \mathrm{kg}$ ) or vehicle (veh) for 2 weeks. Rats were then implanted with a cannula directed into the lateral ventricle, made diabetic with an i.v. injection of streptozotocin $(50 \mathrm{mg} / \mathrm{kg})$, and treated daily with either leptin $(10 \mu \mathrm{g})$ or vehicle (veh). At the end of the experimental period, rats were anesthetized with pentobarbital and the spleens were collected and frozen in liquid nitrogen for subsequent norepinephrine and protein determinations. Results are shown as means \pm s.E.M. Group means represent between 6 and 11 observations. Means with different letters indicate statistically significant differences $(P<0 \cdot 05)$.

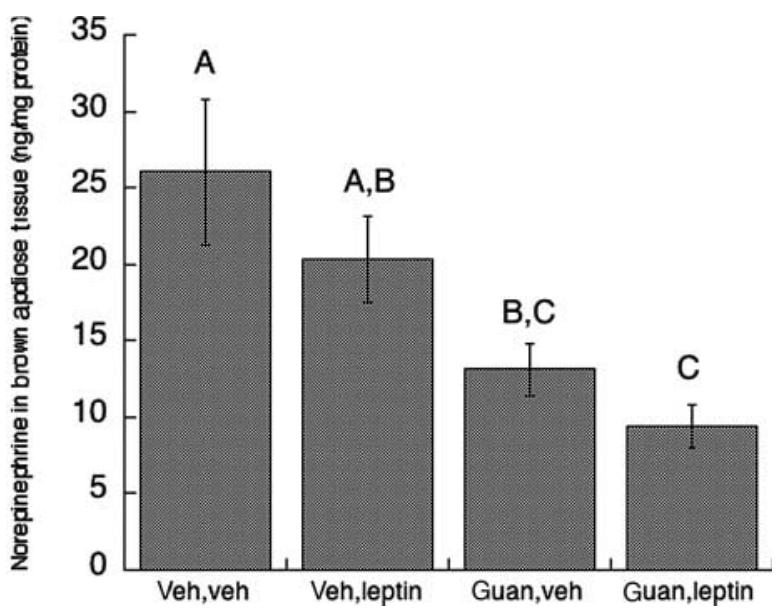

Figure 4 Norepinephrine content in the brown adipose tissue (ng/mg protein). Wistar rats were pretreated every other day with either guanethidine (guan) $(100 \mathrm{mg} / \mathrm{kg}$ ) or vehicle (veh) for 2 weeks. Rats were then implanted with a cannula directed into the lateral ventricle, made diabetic with an i.v. injection of streptozotocin $(50 \mathrm{mg} / \mathrm{kg})$, and treated daily with either leptin $(10 \mu \mathrm{g})$ or vehicle (veh). At the end of the experimental period, rats were anesthetized with pentobarbital and brown adipose tissues were collected and frozen in liquid nitrogen for subsequent norepinephrine and protein determinations. Results are shown as means \pm s.E.M. Group means represent between 6 and 11 observations. Means with different letters indicate statistically significant differences $(P<0 \cdot 05)$.

\section{Discussion}

Our hypothesis that prior guanethidine treatment would block or attenuate the ability of central leptin administration to decrease blood glucose concentrations in diabetic rats was not supported by the results. Both guanethidine-treated and vehicle-treated diabetic rats responded to i.c.v. leptin with an attenuation of blood glucose concentrations. An analysis of the tissue norepinephrine content suggested that the prior guanethidine treatment was effective at suppressing sympathetic activity in most of the tissues examined. Brown adipose tissue, white adipose tissue, and spleen, all showed a guanethidine-related decrease in norepinephrine content. Average norepinephrine content remaining in these tissues of guanethidine-treated rats ranged from 15 to $39 \%$ of control values; however, the norepinephrine content of the liver was not affected by prior guanethidine treatment. This contrasts with the findings of Villanueva et al. (2003) who reported that guanethidine-treated rats have less than 3\% of the liver norepinephrine content of control animals. This difference is most likely accounted for by either differences in the age of the animals when guanethidine was first introduced or in the route of administration or both. In the present study, guanethidine was injected s.c. (subQ) beginning when the rats were just over 3 weeks of age (body weight $\sim 50 \mathrm{~g}$ ),

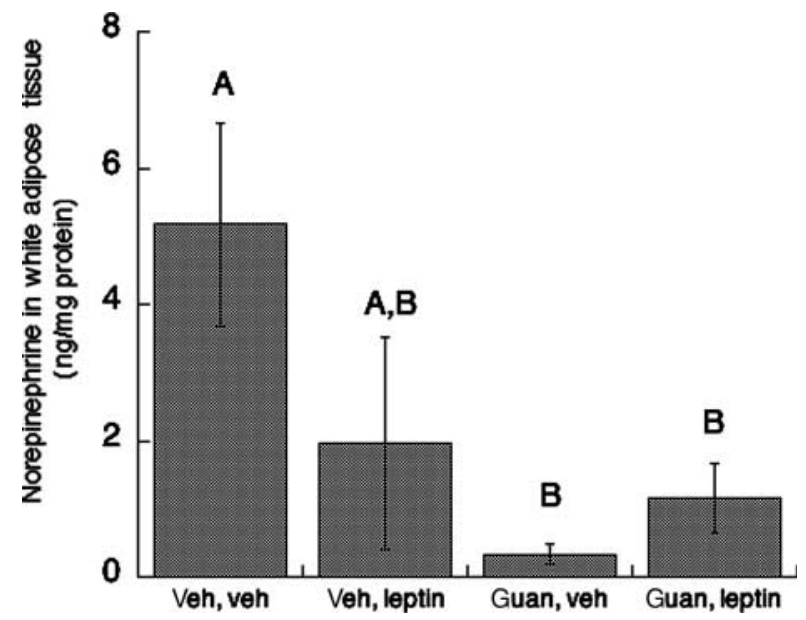

Figure 5 Norepinephrine content in the white adipose tissue (ng/mg protein). Wistar rats were pretreated every other day with either guanethidine (guan) $(100 \mathrm{mg} / \mathrm{kg}$ ) or vehicle (veh) for 2 weeks. Rats were then implanted with a cannula directed into the lateral ventricle, made diabetic with an i.v. injection of streptozotocin $(50 \mathrm{mg} / \mathrm{kg})$, and treated daily with either leptin $(10 \mu \mathrm{g})$ or vehicle (veh). Results are shown as means \pm s.E.M. At the end of the experimental period, rats were anesthetized with pentobarbital and white adipose tissues were collected and frozen in liquid nitrogen for subsequent norepinephrine and protein determinations. Group means represent between 5 and 9 observations, with the exception of the vehicle, leptin group, which had only two observations. Several leptin-treated rats had no visible fat. Means with different letters indicate statistically significant differences $(P<0 \cdot 05)$. 


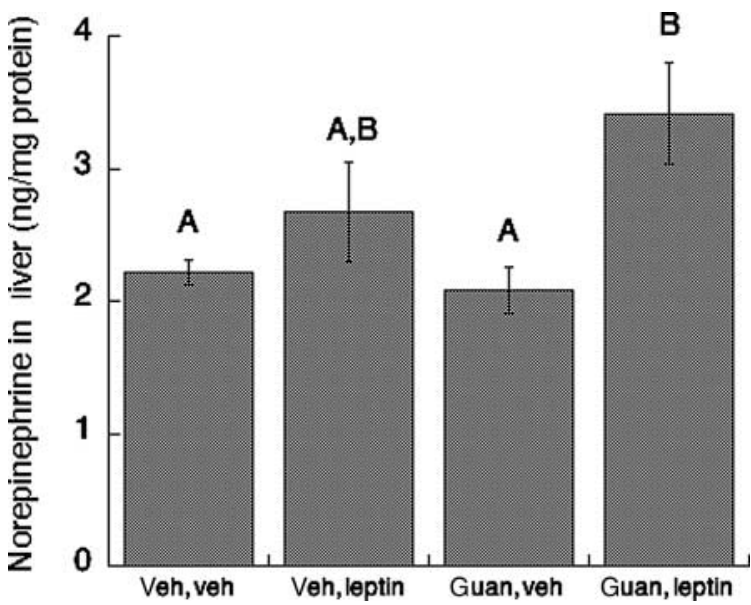

Figure 6 Norepinephrine content in the liver (ng/mg protein). Wistar rats were pretreated every other day with either guanethidine (guan) $(100 \mathrm{mg} / \mathrm{kg}$ ) or vehicle (veh) for 2 weeks. Rats were then implanted with a cannula directed into the lateral ventricle, made diabetic with an i.v. injection of streptozotocin (50 mg/ $/ \mathrm{kg}$ ), and treated daily with either leptin $(10 \mu \mathrm{g})$ or vehicle (veh). At the end of the experimental period, rats were anesthetized with pentobarbital and livers were collected and frozen in liquid nitrogen for subsequent norepinephrine and protein determinations. Results are shown as means \pm s.E.M. Group means represent between 6 and 11 observations. Means with different letters indicate statistically significant differences $(P<0 \cdot 05)$.

whereas Villanueva et al. administered guanethidine via an i.p. route when the rats were 6 days old. Neonatal animals are more sensitive to the effects of guanethidine than older animals (Johnson \& O'Brien 1976) and an i.p. route of administration might be expected to affect the liver more than a subQ route.

There are three possible interpretations of the present data: 1) The sympathetic nervous system does not mediate leptin's ability to normalize blood glucose concentrations in STZinduced diabetic rats, 2) The sympathetic nervous system is involved in leptin's ability to normalize blood glucose concentrations, but only a small fraction of the total sympathetic activity is enough to have the full effect, and 3) The sympathetic nervous system is required to mediate the effects of leptin, but it only involves tissues that are not affected by guanethidine treatment (e.g., liver, as shown herein). Others have found that $\alpha 1, \beta 1, \beta 2$, or $\beta 3$ adrenergic receptor blockade did not affect the ability of centrally administered leptin to normalize blood glucose concentrations in STZ-induced diabetic rats (da Silva et al. 2006). However, the combination of $\alpha 1, \beta 1$, and $\beta 2$ adrenergic receptor blockade did attenuate the leptin-induced rise in heart rate. Therefore, the present results, using guanethidine treatment, and the findings of da Silva et al. (2006), using adrenergic receptor antagonists, both suggest that the sympathetic nervous system is not required for centrally administered leptin to lower the blood glucose concentrations of STZ-induced diabetic rats.
In our previous studies and those of others, chronic central leptin administration normalized blood glucose concentrations of diabetic rats within several days and blood glucose remained at normal levels until the end of the study (Lin et al. 2002, da Silva et al. 2006, White \& Wernette unpublished observations). In the present study, we stopped the daily injections of leptin after day 7 and found that the blood glucose concentrations of the rats previously injected with leptin increased between days 7 and 16, indicating that the blood glucose lowering effect of leptin is reversible. Resuming the daily leptin injections on day 16 once again resulted in a decrease in the blood glucose concentrations of the diabetic rats. To our knowledge, the reversibility of the glucose lowering effect of i.c.v. leptin has not been demonstrated previously.

Guanethidine pretreatment accentuated the ability of leptin to decrease blood glucose concentrations over time. This was not manifested until the last 2 days of the first set of leptin injections (phase 1). However, this accentuation carried over through the period of recovery from leptin injection and throughout the second set of leptin injections (phase 2). Guanethidine treatment in the absence of leptin administration had no effect on blood glucose concentrations. This means that blood glucose concentrations are significantly lower in response to central leptin administration in the absence of a fully functional sympathetic nervous system. This suggests that some aspect of sympathetic nervous activity increases blood glucose concentrations in leptin-treated rats. While it is beyond the scope of this study to address the cause, sympathetic stimulation is thought to increase hepatic glucose production (Nonogaki 2000), which would tend to increase blood glucose concentrations in rats not pretreated with guanethidine. The effect of the sympathetic nervous system on hepatic glucose production appears to be mediated through a combination of direct innervation of the liver, epinephrine from the adrenal medulla, and glucagon from the pancreas (Nonogaki 2000). However, we did not observe a decrease in the norepinephrine content from livers of guanethidine-treated rats in the present study and guanethidine treatment does not affect epinephrine release from the adrenal medulla (Johnson et al. 1975).

In nondiabetic rats, pretreatment with guanethidine has been found to enhance the reduction in food intake caused by leptin by about 20\% (Dobbins et al. 2002). Though food intake was not determined in the present study, it is possible that a decrease in food intake could have accentuated the leptin-induced decrease in the blood glucose concentrations that we found in rats pretreated with guanethidine. However, since this guanethidine-related decrease in blood glucose was observed even after leptin injections had been terminated for 9 days, it could also be related to factors other than a decrease in food intake. It is important to note that the leptin-induced decrease in blood glucose concentrations of diabetic rats is not due to a decrease in food intake. We and others have previously shown that pair-feeding nondiabetic or diabetic rats to the amount eaten by leptin-treated rats does not result 
in decreased blood glucose concentrations (Dobbins et al. 2002, Hidaka et al. 2002, Lin et al. 2002). Thus, while the accentuated decrease in blood glucose concentration due to guanethidine pretreatment may be related to a reduction in food intake, it appears that the ability of leptin to decrease blood glucose concentrations in diabetic rats is not.

The induction of diabetes by STZ causes a decrease in body weight, despite increases in food intake (Sindelar et al. 1999). In the present study, diabetes caused a $40 \mathrm{~g}$ loss of body weight over a period of 3 weeks. Pretreatment with guanethidine, cut the loss in body weight by $\sim 50 \%$ to $22 \mathrm{~g}$. This suggests that activation of the sympathetic nervous system plays a large role in the body weight loss of diabetic rats. Since leptin levels are decreased in STZ-induced diabetic rats (Sindelar et al. 1999), it also suggests that the sympathetic activity-related decrease in body weight cannot be caused by an increase in leptin. Indeed, leptin treatment also tended to attenuate body weight loss in diabetes rats, mostly due to its affect in rats pretreated with guanethidine. The combination of pretreatment with guanethidine and leptin treatment of diabetic rats negated any weight loss. Guanethidine pretreatment of nondiabetic rats has been found to increase the metabolic efficiency after leptin treatment (Dobbins et al. 2002). It was suggested that this is related to altering leptin-stimulated sympathetic activity, which affects substrate utilization.

If the sympathetic nervous system does not mediate the ability of centrally administered leptin to decrease blood glucose concentrations in diabetic rats, what might be the mediator? A brain-liver circuit has been postulated that involves the hypothalamus, brainstem, hepatic branch of the vagus nerve (vagal efferents), and liver (Pocai et al. 2005). Inhibition of fatty acid oxidation in hypothalamic nuclei selectively activates the nucleus of the solitary tract and the dorsal motor nucleus of the vagus nerve in the brainstem: processes that are dependent upon ATP-dependent potassium channels. Activation of these brainstem nuclei leads to inhibition of hepatic gluconeogenesis and glucose production via vagal efferent neurons. It is of interest to note that leptin also activates ATP-dependent potassium channels within selective hypothalamic neurons (Spanswick et al. 1997). Therefore, it is possible that leptin administered into the brain may use a similar pathway to inhibit gluconeogenesis and lower blood glucose concentrations in diabetic rats. If this is the case, leptin may be expected to produce greater vagal tone in the absence of a fully functional sympathetic nervous system. This would result in lower blood glucose concentrations than if the sympathetic nervous system were intact, as observed in the present study.

In conclusion, use of the neurotoxin guanethidine suggests that the sympathetic nervous system does not mediate the ability of central leptin administration to normalize or attenuate the high blood glucose concentrations in STZinduced diabetic rats. This supports the findings of others (da Silva et al. 2006), who used antagonists to the various adrenergic receptors to block the sympathetic nervous system. Further research is needed to elucidate the mechanism by which centrally administered leptin normalizes or attenuates blood glucose concentrations in STZ-induced diabetic rats.

\section{Declaration of interest}

There is no conflict of interest that would prejudice its impartiality.

\section{Funding}

This work was financially supported by a BioGrant from Auburn University.

\section{References}

Andreelli F, Foretz M, Knauf C, Cani PD, Perrin C, Iglesias MA, Pillot B, Bado A, Tronche F, Methieux G et al. 2006 Liver adenosine monophosphate-activated kinase-a2 catalytic subunit is a key target for the control of hepatic glucose production by adiponectin and leptin but not insulin. Endocrinology 147 2432-2441.

Bergeron R, Previs SF, Cline GW, Perret P, Russell RR, Young LH \& Shulman GI 2001 Effect of 5-aminoimidazole-4-carboxamide-1- $\beta$-Dribofuranoside infusion on in vivo glucose and lipid metabolism in lean and obese Zucker rats. Diabetes 50 1076-1082.

Chinookoswong N, Wang J-L \& Shi Z-Q 1999 Leptin restores euglycemia and normalizes glucose turnover in insulin-deficient diabetes in the rats. Diabetes 48 1487-1492.

Dobbins RL, Szczepaniak LS, Zhang W \& McGarry JD 2002 Chemical sympathectomy alters regulation of body weight during prolonged ICV leptin infusion. American Journal of Physiology. Endocrinology and Metabolism 284 E778-E787.

Haque MS, Minokoshi Y, Hamai M, Iwai M, Horiuchi M \& Shimazu T 1999 Role of the sympathetic nervous system and insulin enhancing glucose uptake in peripheral tissues after intrahypothalamic injection of leptin in rats. Diabetes 48 1706-1712.

Haynes WG 2000 Interaction between leptin and sympathetic nervous system in hypertension. Current Hypertension Reports 2 311-318.

Haynes WG, Morgan DA, Walsh SA, Mark AL \& Sivitz WI 1997 Receptormediated regional sympathetic nerve activation by leptin. Journal of Clinical Investigation $100270-278$

Hidaka S, Yoshimatsu H, Kondou S, Tsuruta Y, Oka K, Noguchi H, Okamoto K, Sakino H, Teshima Y, Okeda T et al. 2002 Chronic central leptin infusion restores hyperglycemia independent of food intake and insulin level in streptozotocin-induced diabetic rats. FASEB Journal 16 509-518.

Johnson EM \& O'Brien F 1976 Evaluation of the permanent sympathectomy produced by the administration of guanethidine to adult rats. Journal of Pharmacology and Experimental Therapeutics 196 53-61.

Johnson EM Jr, Cantor E \& Douglas JR Jr 1975 Biochemical and functional evaluation of the sympathectomy produced by the administration of guanethidine to newborn rats. Journal of Pharmacology and Experimental Therapeutics 193 503-512.

Lin C-Y, Higginbotham DA, Judd RL \& White BD 2002 Central leptin increases insulin sensitivity in streptozotocin-induced diabetic rats. American Journal of Physiology. Endocrinology and Metabolism 282 E1084-E1091.

Merrill GF, Kurth EF, Hardie DG \& Winder WW 1997 AICA riboside increases AMP-activated protein kinase, fatty acid oxidation, and glucose uptake in rat muscle. American Journal of Physiology. Endocrinology and Metabolism 272 E1107-E1112.

Minokoshi Y, Kim Y-B, Peroni OD, Fryer LGD, Muller C, Carling D \& Kahn BB 2002 Leptin stimulates fatty-acid oxidation by activating AMP-activated protein kinase. Nature 415 339-343. 
Nonogaki K 2000 New insights into sympathetic regulation of glucose and fat metabolism. Diabetologia 43 533-549.

Pocai A, Obici S, Schwartz GJ \& Rossetti L 2005 A brain-liver circuit regulates glucose homeostasis. Cell Metabolism 1 53-61.

Da Silva AA, Tallam LS, Liu J \& Hall JE 2006 Chronic antidiabetic and cardiovascular actions of leptin: role of CNS and increased adrenergic activity. American Journal of Physiology. Regulatory, Integrative and Comparative Physiology 291 R1275-R1282.

Sindelar DK, Havel PJ, Seeley RJ, Wilkinson CW, Woods SC \& Schwatz MW 1999 Low plasma leptin levels contribute to diabetic hyperphagia in rats. Diabetes 48 1275-1280.

Smith AC, Bruce CR \& Dyck DJ 2005 AMP kinase activation with AICAR simultaneously increases fatty acid and glucose oxidation in resting rat soleus muscle. Journal of Physiology 565.2 537-546.
Spanswick D, Smith MA, Groppi VE, Logan SD \& Ashford ML 1997 Leptin inhibits hypothalamic neurons by activation of ATP-sensitive potassium channels. Nature 390 521-525.

Villanueva I, Pinon M, Quevedo-Corona L, Martinez-Olivares R \& Racotta R 2003 Epinephrine and dopamine colocalization with norepinephrine in various peripheral tissues: guanethidine effects. Life Sciences $\mathbf{7 3}$ 1645-1653.

Received in final form 4 June 2008

Accepted 3 July 2008

Made available online as an Accepted Preprint 3 July 2008 